\title{
Complex Narrow Band-Pass Filters for QRS Detection in Contactless Magnetocardiograms of Small Animals
}

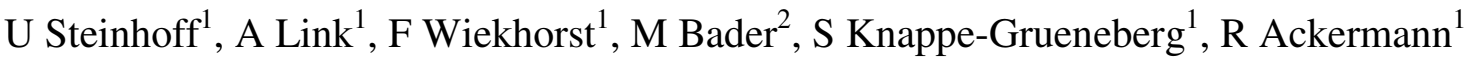 \\ ${ }^{1}$ Physikalisch-Technische Bundesanstalt, Berlin, Germany \\ ${ }^{2}$ Max-Delbrück-Center for Molecular Medicine, Berlin, Germany
}

\begin{abstract}
Recently, magnetocardiography (MCG) of small animals was proposed for the investigation of drug safety and for phenotype characterization of genetically modified mice. MCG is a contactless measurement method to provide information very similar to the ECG. In order to cover the wide variation of signal shape and frequency content of the heart signals of small animals, we used a complex narrow-band filter characterized by center frequency and bandwidth as a preprocessor for QRS detection. We applied the method to MCG signals of rabbits, hamsters, guinea pigs and mice. While in human MCGs the preferential center frequency of the bandpass filter is $20 \mathrm{~Hz}$ at a bandwidth of $2 \mathrm{~Hz}$, the respective values for rabbits are 40(4) $\mathrm{Hz}$, for guinea pigs 40(4) $\mathrm{Hz}$, for hamsters 100(10) $\mathrm{Hz}$, and for mice 150(30) $\mathrm{Hz}$. The method works stable even in cases where the signal to noise ratio is only 2:1.
\end{abstract}

\section{Introduction}

Telemetric ECG measurement is a commonly used method in drug safety assessment and in phenotype characterization of genetically modified animals. An alternative is provided by the contactless measurement of the magnetocardiogram [1]. MCG is the measurement of the magnetic field generated by the same currents that give rise to the ECG. Hence the MCG contains to a large degree information similar to the ECG [2].

The MCG signals of small animals are varying in shape and frequency content depending on species, genetic changes, pharmacological load and stress factors. Since the animals are measured in conscious unrestricted state, the actual shape of the QRS complex might also change in dependence on the animals position relative to the sensors. This makes the use of template matching methods difficult.

However, the QRS complex is always characterized by a strong amplitude variation localized in time. Morlet wavelet filters are appropriate tools to analyze such signals. This paper describes how a bandpass filter derived from the Morlet wavelet can be used as a preprocessor for QRS detection in small animals MCGs.

\section{Methods}

\subsection{Magnetocardiographic measurements}

MCG was measured using two different multi-channel biomagnetometers. One system was custom made and contained sixteen magnetic field sensors (SQUIDs) immersed in liquid helium inside a dewar vessel [3]. The second system was a commercially available 93-SQUID system (Eagle Technology). The conscious and unrestrained animals (guinea pig, rabbit, hamster and mouse) were placed in suitable containers within a distance of approximately $2-3 \mathrm{~cm}$ to the closest sensor, skin contact is not required during MCG [1,4]. All measurements were performed according to the German Animal Welfare Law (Tierschutzgesetz) and with approval of the local ethics committee. Signals were acquired for 60 to $600 \mathrm{~s}$, the sampling rate was between 1 and $3 \mathrm{kHz}$.

\subsection{Narrow band-pass filter}

A complex phase linear bandpass filter $h$ with a Gaussian magnitude response function is used for signal preprocessing [5]. Such a filter has an optimal timebandwidth product suitable for a time frequency analysis.

For the input $x[n](n=0, \ldots, N-1)$ the output $z[n]$ of a complex FIR filter is the following convolution sum:

$$
\begin{aligned}
z[n]= & u[n]+\mathrm{j} v[n] \\
= & (\operatorname{Re}(h) * x)[n]+\mathrm{j}(\operatorname{Im}(h) * x)[n] \\
= & \sum_{k=-L}^{k=L} \operatorname{Re}(h[k]) x[n-k]+ \\
& \mathrm{j} \sum_{k=-L}^{k=L} \operatorname{Im}(h[k]) x[n-k]
\end{aligned}
$$

where $[\mathrm{n}]$ indicates the value at sample point $\mathrm{n}$.

Envelope $E[n]$ and phase $\Phi[n]$ of the output signal $z[n]$ 
are given in terms of the Hilbert transform pair $u[n]$ and $v[n]$ :

$$
E[n]=\sqrt{u^{2}[n]+v^{2}[n]}
$$

and

$$
\Phi[n]=\operatorname{Arctan}\left(\frac{v[n]}{u[n]}\right)
$$

where the multi-branch character of the arctangent function is indicated by the capital A.

In our case, the set of $2 L+1$ complex filter impulse response coefficients $h[k]$ is given by the following equations:

$$
\begin{aligned}
& h[k]=\frac{2 \gamma[k]}{\sum_{k=-L}^{L}|\gamma[k]|} \quad k=-L, \mathrm{~K}, 0, \mathrm{~K} L \\
& \gamma[k]=\exp \left(-\mathrm{j} 2 \pi f_{c} k\right) \exp \left(-\frac{\left(2 \pi \delta_{f} k\right)^{2}}{\ln 4}\right)
\end{aligned}
$$

The real part $h_{r e}[k]$ and the imaginary part $h_{i m}[k]$ of the FIR coefficients act as bandpass filters with a center frequency $f_{c}$ and a bandwidth of $2 \delta_{f}$, both given in fractions of the sampling frequency $f_{s}$. To give an example, if the sampling rate $f_{s}$ is $2 \mathrm{kHz}$, the desired center frequency $f_{c} f_{s}$ is $40 \mathrm{~Hz}$ and the half bandwidth $\delta_{f} f_{s}$ is $8 \mathrm{~Hz}$, equation (5) has to be used with $f_{c}=0.02$ and $\delta_{f}=0.002$.

The frequency-response magnitudes of $h_{r e}[k]$ and $h_{i m}[k]$ are $0 \mathrm{~dB}$ at the center frequency $f_{c}$ and $3 \mathrm{~dB}$ at frequencies $f_{c}+\delta_{f}$ and $f_{c}-\delta_{f}$. Their phase responses are shifted by 90 degrees.

The absolutes of the coefficients $h[n]$ decrease with increasing $L$. We have chosen a sufficient large $L>$ $0.55 / \delta_{f}$, ensuring the absolutes of the filter coefficients $|h[L]|$ to be below $10^{-6}$. For this large number of filter coefficients the stopband attenuation of the magnitude response is greater than $90 \mathrm{~dB}$. In the example given above, 551 filter coefficients have to be used.

\subsection{QRS detection}

The envelope $\mathrm{E}$ of the filter output was calculated from single MCG channels according to equation (2). Due to the properties of the bandpass filter, the resulting signal is always positive, it is smooth and it displays high amplitudes only during the QRS complexes (see lower trace in figure 1). In cases of poor signal-to-noise ratio, e.g. in the mouse MCG, the envelope signal of several channels was averaged.

A simple threshold is introduced on the envelope signal $\mathrm{E}$ and the local maxima larger than the threshold are identified as QRS complexes.

\section{Results}

\subsection{Adapted filter parameters}

In order to determine adapted filter parameters for the different species, we performed a wavelet analysis of the respective MCG signals. The result is shown in figure 2.
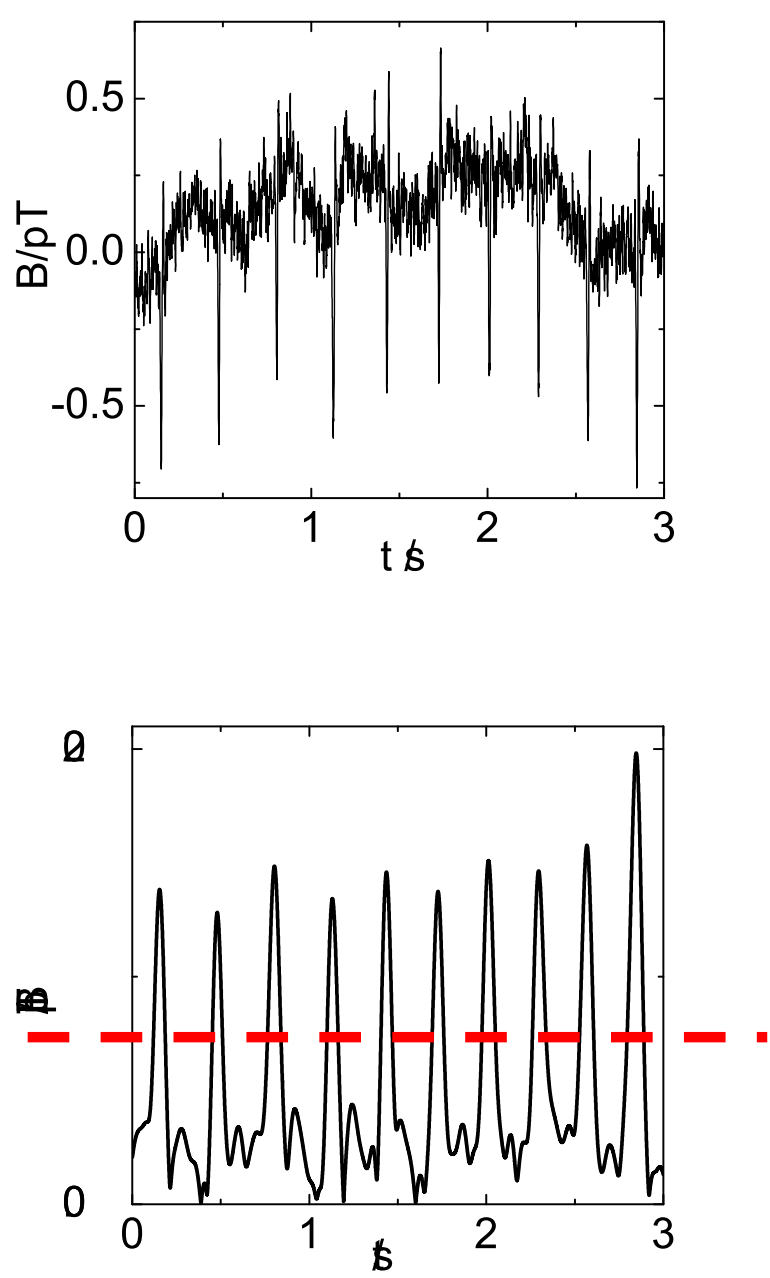

Figure 1. The upper trace shows one channel of a mouse MCG. The lower trace shows the envelope signal $\left(f_{c} f_{s}=150 \mathrm{~Hz}, \delta_{f} f_{s}=30 \mathrm{~Hz}\right)$. Local maxima exceeding the threshold (dashed line) imply an occurrence of a QRS complex. 

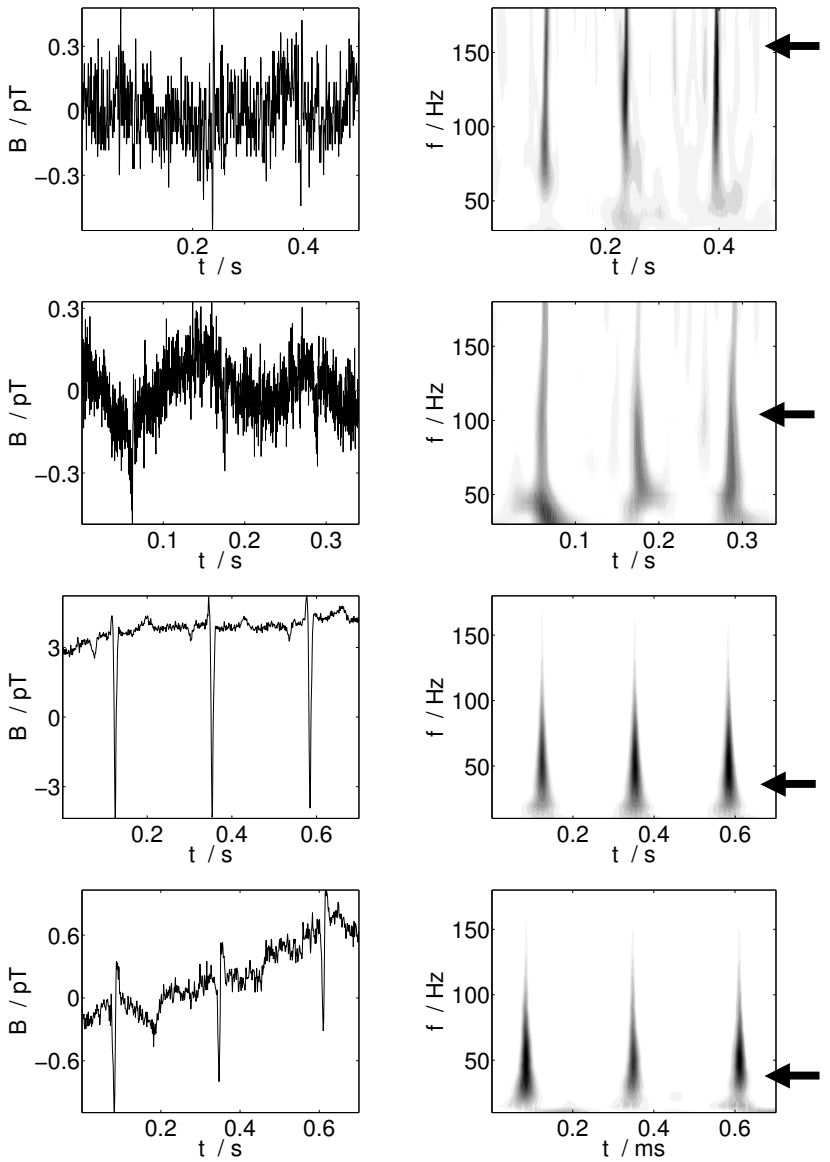

Figure 2. Left side from top to bottom: One MCG channel from a mouse, a hamster, a guinea pig and a rabbit. Right side: wavelet analysis of the left side signals. Dark values imply high energy in the given time-frequency window; the arrows indicate the chosen center frequency for that species (see table 1).

Table 1. Filter center frequencies and bandwidth for different species.

\begin{tabular}{lll} 
Species & $\begin{array}{l}\text { Center frequency } \\
f_{c} f_{s} / \mathrm{Hz}\end{array}$ & $\begin{array}{l}\text { Half Bandwidth } \\
\delta_{f} f_{s} / \mathrm{Hz}\end{array}$ \\
\hline Mouse & 150 & 30 \\
Hamster & 100 & 10 \\
Guinea pig & 40 & 4 \\
Rabbit & 40 & 4
\end{tabular}

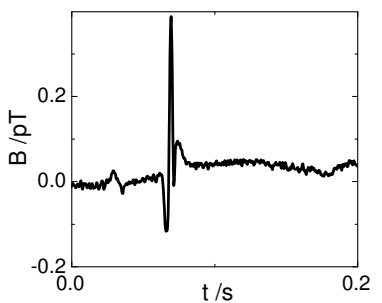

mouse

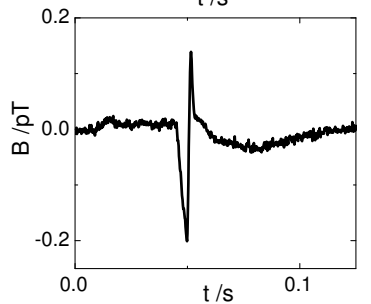

hamster

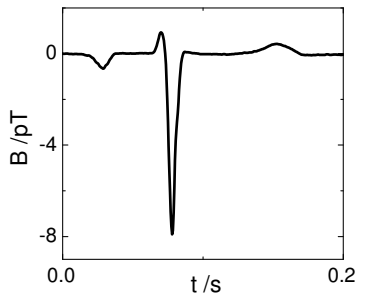

guinea pig

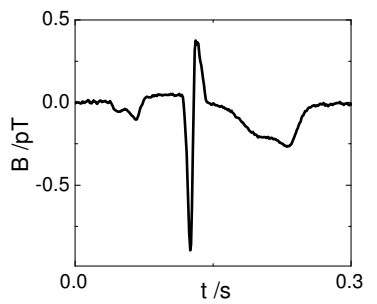

rabbit

Figure 3. Averaged MCG signals. from top to bottom: One MCG channel from a mouse, a hamster, a guinea pig and a rabbit. Quantitative assessment of P-, QRS- and Twave is possible.

It can be seen that the signal-to-noise ratio differs over frequency bands. We excluded frequencies lower than $30 \mathrm{~Hz}$ from the display, since the signal-to-noise ratio was always poor in that frequency range. However, between 30 and $200 \mathrm{~Hz}$ the QRS complexes are clearly detectable.

Preferential values of filter parameters for the different species are summarized in table 1 . It can be argued, that the best detection is possible, if the filter center frequency matches the main frequency content of the QRS complex.

Since the actual QRS shape is of less importance, the methods works even in cases when the animals are moving below the sensors and the QRS template changes considerably.

The bandwidth has to be limited in order to exclude strong noise influences, especially from animal movement, breathing and muscle noise. 


\subsection{Application to signal averaging and beat-to-beat interval detection}

After using the described QRS detection method, we were able to construct averaged heart beats. In some cases, a clustering algorithm provided a further distinction of the identified QRS complexes. The results for the signals from figure 2 are shown in figure 3 . The signal quality improved in all cases, so that the quantitative assessment of P-, QRS- and T-wave became possible.

The calculation of beat-to-beat intervals also allows the study of a number of physiological parameters. An example for beat-to-beat variation of the RR-interval of a conscious unrestrained mouse is given in figure 4 .

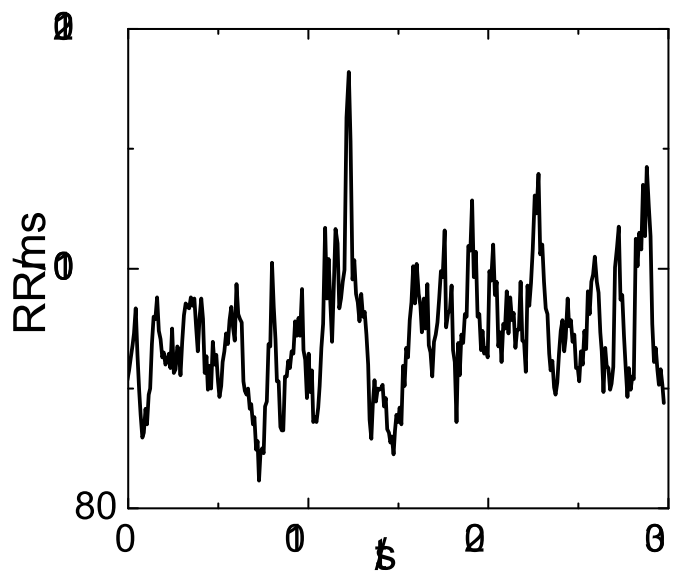

Figure 4. RR intervals determined from the MCG of a conscious unrestrained mouse.

\section{Discussion and conclusions}

Narrow band-pass filters based on a modified Morlet wavelet are suitable preprocessors for QRS detection in the MCG of small animals. By variation of center frequency and filter bandwidth, the method accounts for differences caused by species, pathological changes or pharmacological load. It gives acceptable results even in cases of poor signal-to-noise ratio and changing position of the animals. The method will be useful in the design of high throughput systems for characterization of small animals electrophysiology [6].

\section{Acknowledgements}

This work was partially supported by DFG grant KO2869/1-1 and BMBF grant FKZ-13N8535.

\section{References}

[1] Steinhoff U, Knappe-Grueneberg S, Schnabel A, Trahms L, Smith F, Langley P, et al. Magnetocardiography for pharmacology safety studies requiring high patient throughput and reliability. J Electrocardiol 2004;37 Suppl:187-92.

[2] Fenici R, Brisinda D, Meloni AM. Clinical application of magnetocardiography. Expert Rev Mol Diagn 2005;5(3):291-313.

[3] Burghoff M, Schnabel A, Drung D, Thiel F, KnappeGruneberg S, Hartwig S, et al. Discrimination of multiple sources using a SQUID vector magnetometer. Neurol Clin Neurophysiol 2004:67.

[4] Steinhoff U, Wilhelm C, Wiekhorst F, Lee SY, Ackermann $\mathrm{R}$, Bader $\mathrm{M}$, et al. Contactless magnetocardiographic characterization of knock-out mice. Folia Cardiologica 2005;12(suppl.D):396-8.

[5] Link A, Endt P, Oeff M, Trahms L. Variability of the QRS signal in high-resolution electrocardiograms and magnetocardiograms. IEEE Trans Biomed Eng 2001;48(2):133-42.

[6] Bauer S, Weber Dos Santos R, Steinhoff U, Wiekhorst F, Ackermann R, Schurig T, et al. Bidomain modelling of electrical activity of the mouse heart supports the design of a specialized magnetocardiographic device. Biomed Tech (Berl) 2005;50(1/1):171-2.

Address for correspondence

Uwe Steinhoff

Physikalisch-Technische Bundesanstalt

Abbestr. 2-12

10587 Berlin

Germany

e-mail: uwe.steinhoff@ptb.de 